Draft Version SEPTEMber 11, 2018

Preprint typeset using $\mathrm{LT}_{\mathrm{E}} \mathrm{X}$ style emulateapj v. 5/2/11

\title{
THE ROLE OF ICE COMPOSITIONS FOR SNOWLINES AND THE C/N/O RATIOS IN ACTIVE DISKS
}

\author{
Ana-Maria A. Piso ${ }^{1,2}$, Jamila Pegues ${ }^{1,3}$, Karin I. ÖBerg $^{1}$ \\ Draft version September 11, 2018
}

\begin{abstract}
The elemental compositions of planets define their chemistry, and could potentially be used as beacons for their formation location if the elemental gas and grain ratios of planet birth environments, i.e. protoplanetary disks, are well understood. In disks, the ratios of volatile elements, such as $\mathrm{C} / \mathrm{O}$ and $\mathrm{N} / \mathrm{O}$, are regulated by the abundance of the main $\mathrm{C}, \mathrm{N}, \mathrm{O}$ carriers, their ice binding environment, and the presence of snowlines of major volatiles at different distances from the central star. We explore the effects of disk dynamical processes, molecular compositions and abundances, and ice compositions on the snowline locations of the main $\mathrm{C}, \mathrm{O}$ and $\mathrm{N}$ carriers, and the $\mathrm{C} / \mathrm{N} / \mathrm{O}$ ratios in gas and dust throughout the disk. The gas-phase $\mathrm{N} / \mathrm{O}$ ratio enhancement in the outer disk (exterior to the $\mathrm{H}_{2} \mathrm{O}$ snowline) exceeds the $\mathrm{C} / \mathrm{O}$ ratio enhancement for all reasonable volatile compositions. Ice compositions and disk dynamics individually change the snowline location of $\mathrm{N}_{2}$, the main nitrogen carrier, by a factor of 2-3, and when considered together the range of possible $\mathrm{N}_{2}$ snowline locations is $\sim 11-\sim 79$ AU in a standard disk model. Observations that anchor snowline locations at different stages of planet formation are therefore key to develop $\mathrm{C} / \mathrm{N} / \mathrm{O}$ ratios as a probe of planet formation zones.
\end{abstract}

\section{INTRODUCTION}

The chemical composition of protoplanetary disks is largely dictated by the freeze-out of volatile species. The snowline locations of volatile molecules are therefore crucial in determining disk chemical abundances in gas and dust, as well as planet compositions.

Carbon and oxygen bearing molecules, such as $\mathrm{H}_{2} \mathrm{O}$, $\mathrm{CO}_{2}$ and $\mathrm{CO}$, as well as the carbon-to-oxygen $(\mathrm{C} / \mathrm{O})$ ratio in protoplanetary disks and in giant planet atmospheres have been extensively studied from a theoretical standpoint (Öberg et al. 2011b, Ali-Dib et al. 2014, Madhusudhan et al. 2014, Mollière et al. 2015), and snowlines of volatiles such as $\mathrm{H}_{2} \mathrm{O}$ and $\mathrm{CO}$ have been detected (Zhang et al. 2013, Qi et al. 2013). However, disk chemistry involves many other molecular compounds (Henning \& Semenov 2013) including nitrogen bearing species and hydrocarbons (e.g., Mandell et al. 2012), which may affect the compositions of nascent planets.

Both in Solar system comets and in protoplanetary disks, volatile carbon and oxygen are primarily contained in $\mathrm{H}_{2} \mathrm{O}, \mathrm{CO}_{2}$ and $\mathrm{CO}$ (e.g., Lodders 2003, Mumma \& Charnley 2011, Öberg et al. 2011b, Boogert et al. 2015). However, some fraction of carbon may also be carried by $\mathrm{CH}_{4}$ (e.g., Öberg et al. 2008), which may change the $\mathrm{C} / \mathrm{O}$ ratio in gas and in dust at some disk locations. In the case of nitrogen, chemical models of the protostellar nebula (e.g., Owen et al. 2001) and of protoplanetary disks (e.g., Rodgers \& Charnley 2002) suggest that $\mathrm{N}_{2}$ was the dominant form of nitrogen, and that giant planets have accreted their nitrogen content primarily as $\mathrm{N}_{2}$ (Mousis et al. 2014). Due to the high volatility of $\mathrm{N}_{2}$, the gas phase nitrogen-to-oxygen (N/O) ratio in

\footnotetext{
${ }^{1}$ Harvard-Smithsonian Center for Astrophysics, 60 Garden Street, Cambridge, MA 02138

${ }^{2}$ UCLA, 595 Charles E. Young Drive East, Los Angeles, CA 90095

${ }^{3}$ Department of Astrophysical Sciences, Princeton University, Princeton, NJ 08544
}

the outer disk is expected to be high, perhaps more enhanced than the $\mathrm{C} / \mathrm{O}$ ratio compared to the Solar value. The $\mathrm{C} / \mathrm{O}$ ratio in gas cannot exceed unity (i.e., a factor of $\sim 2$ enhancement compared to the Solar value) since the major volatile carbon carrier is CO. In contrast, the $\mathrm{N} / \mathrm{O}$ ratio mainly depends on the relative depletion of $\mathrm{N}_{2}$ and oxygen carriers, and it will increase as each of the oxygen carrier snowlines $\left(\mathrm{H}_{2} \mathrm{O}, \mathrm{CO}_{2}, \mathrm{CO}\right)$ is crossed. Beyond the $\mathrm{CO}$ snowline, there is no strict upper limit to the $\mathrm{N} / \mathrm{O}$ ratio. The spatial extent of this latter region depends on the relative bond strengths of $\mathrm{CO}$ and $\mathrm{N}_{2}$ to ice, but may be quite large (see Section 3). Giant planets that form at wide separations should thus have an excess of nitrogen in their atmospheres, which could be used to trace their formation origin. In addition to $\mathrm{N}_{2}$, a fraction of the nitrogen abundance may also be carried by less volatile species such as $\mathrm{NH}_{3}$ (Bottinelli et al. 2010, Mumma \& Charnley 2011). The present day $\mathrm{N}_{2}$ in Titan's atmosphere, for example, is thought to originate from accretion of primordial $\mathrm{NH}_{3}$ (Atreya et al. 1978 . Mandt et al. 2014).

The snowline locations of the main carbon, oxygen and nitrogen carriers strongly depend on the ice grain composition. Very volatile species, such as $\mathrm{CO}$ and $\mathrm{N}_{2}$, present binding energies, and therefore snowline locations, that are sensitive to the details of the composition of the icy grain mantles. Spectroscopic observations suggest that up to $90 \%$ of the CO ice is frozen in a layer that is thick enough and separated from the $\mathrm{H}_{2} \mathrm{O}$ ice layer underneath so that it can be considered pure ice (e.g., Pontoppidan et al. 2003). However, if the $\mathrm{CO}$ (or $\mathrm{N}_{2}$ ) ice layer is thin enough ( monolayer coverage), then it will interact with the $\mathrm{H}_{2} \mathrm{O}$ ice substrate (e.g., Collings et al. 2003). The ice binding energy is significantly larger in this water dominated environment than in the pure ice case, as shown by laboratory experiments (Collings et al. 2003. Öberg et al. 2005, Bisschop et al. 2006, Fayolle et al. 2016). This implies that ices in different environments 
will sublimate at different radii, which will substantially change the disk regions where these volatiles are present in gaseous or solid form (see Section 3.2). In protostellar cores, $\mathrm{H}_{2} \mathrm{O}$ ice is primarily amorphous e.g., Williams \& Herbst 2002, van Dishoeck et al. 2014). When temperatures exceed $\sim 80-90 \mathrm{~K}, \mathrm{H}_{2} \mathrm{O}$ ice acquires a crystalline structure (Schegerer \& Wolf 2010). The CO binding energy is larger in an amorphous porous $\mathrm{H}_{2} \mathrm{O}$ ice environment than in the amorphous compact or crystalline cases (Noble et al. 2012, Fayolle et al. 2016). The $\mathrm{N}_{2}$ binding energy is also larger for a porous versus compact $\mathrm{H}_{2} \mathrm{O}$ ice substrate. No equivalent studies have yet been performed for the deposition of $\mathrm{N}_{2}$ on crystalline $\mathrm{H}_{2} \mathrm{O}$ ice, but we expect the $\mathrm{N}_{2}$ binding energy in this environment to follow the same trend as the CO binding energy, as $\mathrm{CO}$ and $\mathrm{N}_{2}$ display a similar desorption behavior. To explore the range of distances at which $\mathrm{CO}$ and $\mathrm{N}_{2}$ in different environments desorb, we consider the limiting cases: pure ices (lowest binding energy) and ices residing on an amorphous porous $\mathrm{H}_{2} \mathrm{O}$ ice substrate (highest binding energy). We refer to the latter simply as water dominated ices unless noted otherwise.

In this work, we expand the coupled drift-desorption model developed in (Piso et al. 2015; hereafter Paper I) by considering additional volatile molecules and abundances, ice compositions, as well as nitrogen-to-oxygen $(\mathrm{N} / \mathrm{O})$ ratios. This paper is organized as follows. In Section 2 we review the drift-desorption model developed in Paper I. We discuss the effect of different abundances of the main carbon, oxygen and nitrogen carriers, grain compositions and disk dynamics on snowline locations and the $\mathrm{C} / \mathrm{N} / \mathrm{O}$ ratios in Section 3. We address the implications of our results in Section 4 and summarize our findings in Section 5 .

\section{COUPLED DRIFT-DESORPTION MODEL}

We begin with a brief review of Paper I's model for the effect of radial drift and viscous gas accretion on volatile snowline locations. We review our disk model in Section 2.1. and summarize our numerical method and results in Section 2.2

\subsection{Disk Model}

In this work we consider both a static and a viscous disk. The static disk is irradiated by the central star and does not experience redistribution of solids or radial movement of the nebular gas. To quantify the effects of radial drift and gas accretion, we use a viscous disk with a spatially and temporally constant mass flux, $\dot{M}$. The viscous disk takes into account radial drift, gas accretion onto the central star, as well as accretion heating. We focus on this disk model which includes all the dynamical and thermal processes we are interested in for the scope of this paper, and do not further consider the other disk models presented in Paper I.

Following Chiang \& Youdin (2010), the temperature profile for a static disk is

$$
T_{\text {irr }}=120(r / \mathrm{AU})^{-3 / 7} \mathrm{~K},
$$

where $r$ is the semimajor axis. We use the Shakura \& Sunyaev (1973) steady-state disk solution to model the viscous disk. From Paper I, the viscous disk temperature profile is computed as

$$
T_{\mathrm{visc}}^{4}=\left[\frac{1}{4 r}\left(\frac{3 G \kappa_{0} \dot{M}^{2} M_{*} \mu m_{\mathrm{p}} \Omega_{\mathrm{k}}}{\pi^{2} \alpha k_{\mathrm{B}} \sigma}\right)^{1 / 3}\right]^{4}+T_{\mathrm{irr}}^{4} .
$$

Here $G$ is the gravitational constant, $\kappa_{0}=2 \times 10^{-6}$ is a dimensionless opacity coefficient, $M_{*}=M_{\odot}$ is the mass of the central star, $\mu=2.35$ is the mean molecular weight of the nebular gas, $m_{\mathrm{p}}$ is the proton mass, $\Omega_{\mathrm{k}}=\sqrt{G M_{\odot} / r^{3}}$ is the Keplerian angular velocity, $\alpha=0.01$ is a dimensionless coefficient (see below for details), $k_{\mathrm{B}}$ is the Boltzmann constant, and $\sigma$ is the Stefan-Boltzmann constant.

The steady-state disk has an $\alpha$-viscosity prescription, where the kinematic viscosity is $\nu=\alpha c H$. Here $c \equiv$ $\sqrt{k_{\mathrm{B}} T_{\text {visc }} /\left(\mu m_{\mathrm{p}}\right)}$ is the isothermal sound speed and $H \equiv$ $c / \Omega_{\mathrm{k}}$ is the disk scale height. We can then determine the gas surface density for a viscous disk as (Shakura \& Sunyaev 1973 see also Paper I for a more detailed explanation of these calculations):

$$
\Sigma=\frac{\dot{M}}{3 \pi \nu} .
$$

We choose $\dot{M}=10^{-8} M_{\odot} \mathrm{yr}^{-1}$, consistent with mass flux observations in disks (e.g., Andrews et al. 2010). As described in Paper I, the mass flux rate $M$ and stellar luminosity $L_{*}$ will vary throughout the disk lifetime (Kennedy et al. 2006, Chambers 2009), in contrast with our simplified model which assumes that both quantities are constant. This effect will be most pronounced in the inner disk $(\lesssim$ few $\mathrm{AU})$, where accretion heating dominates. We thus acknowledge that the location of the $\mathrm{H}_{2} \mathrm{O}$ snowline may be determined by the decline in $\dot{M}$ or $L_{*}$ with time, rather than radial drift (see Paper I, Section 2.1 for a more detailed explanation).

\subsection{Desorption-Drift Equations and Results}

The model is described in full in Paper I, here we review and summarize key concepts and results. For a range of initial icy grain sizes composed of a single volatile, we showed in Paper I that the timescale on which these particles desorb is comparable to their radial drift time, as well as to the accretion timescale of the nebular gas onto the central star. We thus have to take into account both drift and gas accretion when we calculate the disk location at which a particle desorbs, since that location may be different from the snowline position in a static disk for a given volatile (see Figure 1 and Öberg et al. 2011b). We determine a particle's final location in the disk by solving the following coupled differential equations:

$$
\begin{aligned}
& \frac{d s}{d t}=-\frac{3 \mu_{x} m_{\mathrm{p}}}{\rho_{\mathrm{s}}} N_{x} R_{\mathrm{des}, \mathrm{x}} \\
& \frac{d r}{d t}=\dot{r},
\end{aligned}
$$

where $s$ is the particle size, $t$ is time, $\mu_{x}$ is the mean molecular weight of volatile $x, \rho_{\mathrm{s}}=2 \mathrm{~g} \mathrm{~cm}^{-3}$ is the density of an icy particle, $N_{x} \approx 10^{15}$ sites $\mathrm{cm}^{-2}$ is the number of adsorption sites of molecule $x$ per $\mathrm{cm}^{-2}, R_{\mathrm{des}, \mathrm{x}}$ is the desorption rate of species $x$, and $\dot{r}$ is the particle's radial drift velocity. We calculate $R_{\text {,des }}$ and $\dot{r}$ as follows. 
The desorption rate $R_{\mathrm{des}, \mathrm{x}}$ (per molecule) is (Hollenbach et al. 2009)

$$
R_{\mathrm{des}, \mathrm{x}}=\nu_{x} \exp \left(-E_{x} / T_{\text {grain }}\right),
$$

where $E_{x}$ is the adsorption binding energy in units of Kelvin, $T_{\text {grain }}$ is the grain temperature (assumed to be the same as the disk temperature, see Paper I), and $\nu_{x}=1.6 \times 10^{11} \sqrt{\left(E_{x} / \mu_{x}\right)} \mathrm{s}^{-1}$ is the molecule's vibrational frequency in the surface potential well. We discuss our choices for $E_{x}$ for the different volatile species in Section 3.1.

Following Chiang \& Youdin (2010) and Birnstiel et al. (2012), a particle's radial drift velocity can be approximated as

$$
\dot{r} \approx-2 \eta \Omega_{\mathrm{k}} r\left(\frac{\tau_{\mathrm{s}}}{1+\tau_{\mathrm{s}}^{2}}\right)+\frac{\dot{r}_{\mathrm{gas}}}{1+\tau_{\mathrm{s}}^{2}},
$$

where the first term is the drift velocity in a non-accreting disk and the second term accounts for the radial movement of the gas. Here $\eta \approx c^{2} /\left(2 v_{\mathrm{k}}^{2}\right)$, where $v_{\mathrm{k}}$ is the Keplerian velocity, and $\tau_{\mathrm{s}} \equiv \Omega_{\mathrm{k}} t_{\mathrm{s}}$ is the dimensionless stopping time:

$t_{\mathrm{s}}= \begin{cases}\rho_{\mathrm{s}} s /(\rho c), & s<9 \lambda / 4 \quad \text { Epstein drag } \\ 4 \rho_{\mathrm{s}} s^{2} /(9 \rho c \lambda), & s<9 \lambda / 4, \text { Re } \lesssim 1 \text { Stokes drag }\end{cases}$

where $\rho$ is the disk mid-plane density, $\lambda$ is the mean free path and Re is the Reynolds number. The gas accretion velocity $\dot{r}_{\text {gas }}$ is determined from $\dot{M}=-2 \pi r \dot{r}_{\text {gas }} \Sigma$, for a fixed $\dot{M}$ and with $\Sigma$ given by Equation 3 .

For a particle of initial size $s_{0}$, we solve the Equation set (4) with the initial conditions $s\left(t_{0}\right)=s_{0}$ and $r\left(t_{0}\right)=r_{0}$, where $t_{0}$ is the time at which we start the integration and $r_{0}$ is the particle's initial location. We stop our simulation after $t_{\mathrm{d}}=3 \mathrm{Myr}$, the disk lifetime, since this is roughly the timescale on which planets form, and determine the desorption timescale $t_{\mathrm{des}}$ from $s\left(t_{\mathrm{des}}\right)=0$, and thus a particle's desorption distance $r_{\mathrm{des}}=r\left(t_{\mathrm{des}}\right)$. Our results are insensitive to our choice of $t_{0}$ as long as $t_{0} \ll t_{\mathrm{d}}$. We note that a particle's size is initially fixed and only changes due to desorption. We thus do not take into account processes such as grain coagulation or fragmentation, which nonetheless occur in disks (e.g., Birnstiel et al. 2012, Pérez et al. 2012). We discuss the effect of these processes on snowline locations in Paper I.

As we show in Paper I, a particle of initial size $s_{0}$ can experience three outcomes after $t_{\mathrm{d}}=3 \mathrm{Myr}$ : (1) it can remain at its initial location, (2) it can drift towards the host star, then stop without evaporating significantly, and (3) it can completely desorb on a timescale shorter than 3 Myr. Particles in scenarios (1) and (2) are thus not affected by radial drift or gas accretion, and the snowline locations are those for a static disk. In contrast, the grains in case (3) desorb practically instantaneously and at a fixed particle-size dependent location in the disk, regardless of their initial position. The snowline locations for these particles will thus be fixed for a given initial particle size and disk model. We have found that grains with sizes $\sim 0.001 \mathrm{~cm} \lesssim s \lesssim 7 \mathrm{~m}$ satisfy this condition for our fiducial disk.

\section{RESULTS}

\subsection{Snowlines in a Static Disk: The Importance of Ice Compositions}

As we note in Section 1, the disk volatile composition and the ice composition determine the location of important snowlines. In this work we focus on the primary carbon, oxygen and nitrogen carriers, i.e. $\mathrm{H}_{2} \mathrm{O}$, $\mathrm{CO}_{2}, \mathrm{CO}, \mathrm{N}_{2}$, and to a lesser extent, $\mathrm{CH}_{4}$ and $\mathrm{NH}_{3}$. Our standard model is based on the median ice abundances observed toward Solar-type protostars (Öberg et al. 2011a), which are $n_{\mathrm{CO}_{2}}=0.29 \times n_{\mathrm{H}_{2} \mathrm{O}}, n_{\mathrm{CO}}=$ $0.38 \times n_{\mathrm{H}_{2} \mathrm{O}}, n_{\mathrm{CH}_{4}}=0.0555 \times n_{\mathrm{H}_{2} \mathrm{O}}$ (hereafter $\mathrm{CH}_{4}$-mid) and $n_{\mathrm{NH}_{3}}=0.055 \times n_{\mathrm{H}_{2} \mathrm{O}}$ (hereafter $\mathrm{NH}_{3}$-mid). Here $n_{\mathrm{H}, \mathrm{O}} \approx 10^{-4} \times n_{\mathrm{H}}$ is the total water abundance (van Dishoeck 2006), with $n_{\mathrm{H}}$ the hydrogen abundance in the disk midplane. For CO, we also take into account that the observed $\mathrm{CO}$ ice only traces some of the $\mathrm{CO}$ reservoir due to its high volatility, and similarly to Öberg et al. (2011b) and Paper I we set the total CO abundance to $0.9 \times 10^{-4} n_{\mathrm{H}}$. Finally, we assume that all nitrogen not found in $\mathrm{NH}_{3}$ is in $\mathrm{N}_{2}$ and assume a Solar nitrogen abundance, $n_{\mathrm{N}}=8 \times 10^{-5} n_{\mathrm{H}}$ (Lodders 2003). In effect, this model assumes no chemical evolution between the protostellar and disk midplane stages. This is reasonable for material that accretes onto the disk at large radii (Visser et al. 2009), but may overestimate the contribution of the original volatiles to the total volatile budget in the innermost disk.

We determine the location of the $\mathrm{H}_{2} \mathrm{O}, \mathrm{CO}_{2}, \mathrm{CO}, \mathrm{CH}_{4}$, $\mathrm{N}_{2}$ and $\mathrm{NH}_{3}$ snowlines in our static disk by balancing desorption with readsorption, following Hollenbach et al. (2009). The binding energies of $\mathrm{H}_{2} \mathrm{O}, \mathrm{CO}_{2}, \mathrm{CO}, \mathrm{CH}_{4}, \mathrm{~N}_{2}$ and $\mathrm{NH}_{3}$ as pure ices are $5800 \mathrm{~K}, 2000 \mathrm{~K}, 834 \mathrm{~K}, 1300$ $\mathrm{K}, 767 \mathrm{~K}$ and $2965 \mathrm{~K}$, respectively (Fraser et al. 2001, Collings et al. 2004, Fayolle et al. 2016, Garrod \& Herbst 2006, Martín-Doménech et al. 2014). For our fiducial disk model, these energies correspond to disk temperatures of $143 \mathrm{~K}, 48 \mathrm{~K}, 21 \mathrm{~K}, 30 \mathrm{~K}, 18 \mathrm{~K}$ and $68 \mathrm{~K}$, respectively. For $\mathrm{CO}$ and $\mathrm{N}_{2}$ as water dominated ices, the binding energies are $1388 \mathrm{~K}$ and $1266 \mathrm{~K}$, respectively (Fayolle et al. 2016), corresponding to disk temperatures of $34 \mathrm{~K}$ and $31 \mathrm{~K}$, respectively. Figure 1 shows the resulting snowline locations, assuming $\mathrm{CO}$ and $\mathrm{N}_{2}$ pure ices (top panel), and $\mathrm{CO}$ and $\mathrm{N}_{2}$ in water dominated ices (bottom panel). The ordinate displays the total carbon, oxygen and nitrogen abundance in solids as a function of the hydrogen total abundance. As expected, the total grain abundance increases with semimajor axis, as more and more species freeze out. Freeze-out at the $\mathrm{CO}_{2}$ and $\mathrm{CO}$ snowlines pulls more heavy elements into the grains than in the case of the $\mathrm{H}_{2} \mathrm{O}$ snowline.

Figure 1, bottom panel, displays the snowline locations when $\mathrm{CO}$ and $\mathrm{N}_{2}$ are in an amorphous porous water environment (see Section 1). The snowlines move outward by a factor of $\sim 3.3$ if the ices are pure, and by up to a factor of $\sim 2$ for an amorphous compact water substrate (not shown; our estimates are based on the results of Fayolle et al. 2016). The CO snowline moves outward by a factor of $\sim 1.5$ if $\mathrm{CO}$ is in a crystalline rather than an amorphous porous water environment (not shown; our estimates are based on the results of Noble et al. 2012), and a similar trend is expected for the $\mathrm{N}_{2}$ snowline (see Section 1 ). The results of Figure 1 thus represent the limiting cases for the positions of the $\mathrm{CO}$ and $\mathrm{N}_{2}$ snowlines, for differ- 


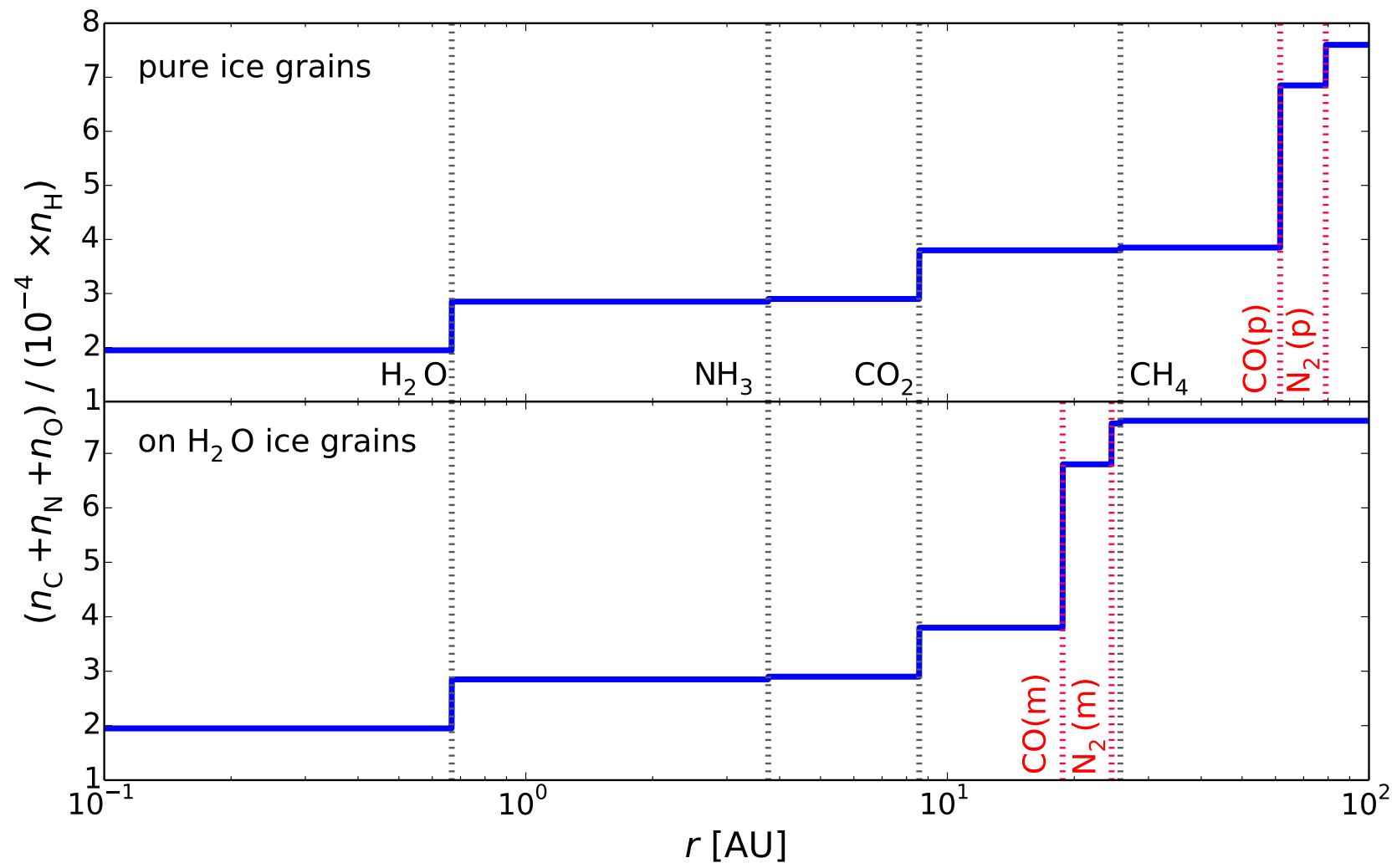

FIG. 1. - The total carbon, nitrogen and oxygen abundance in solids as a function of semimajor axis in a static disk, for CO and $\mathrm{N}_{2}$ as pure ices (top panel) and water dominated ices (bottom panel). Relevant volatile snowlines are marked by the vertical dashed lines. The grain abundances are calculated as a function of the observed median $\mathrm{CH}_{4}$ and $\mathrm{NH}_{3}$ abundances in protostellar cores. The total grain abundance increases with semimajor axis as more and more species freeze out.

ent compositions of the icy grains and morphology of the water ice substrate. This variation in snowline locations changes the chemical abundances both in gas and dust throughout the disk, directly affecting the compositions of nascent giant planets forming in situ. In our simple model, we ignore the effects of $\mathrm{CO}$ and $\mathrm{N}_{2}$ entrapment in water ice through clathrate formation or other processes. Theoretical models aimed at explaining the composition of comet $67 \mathrm{P} /$ Churyumov-Gerasimenko suggest that a small fraction of the total $\mathrm{CO}$ and $\mathrm{N}_{2}$ reservoir may be trapped in clathrates, and only released upon water sublimation (Lectez et al. 2015, Mousis et al. 2016). In this case, the $\mathrm{CO}$ and $\mathrm{N}_{2}$ snowlines would be closer to the star than in the pure ice case.

\section{2. $C / N / O$ Ratios in Static Disks}

In this section we determine the $\mathrm{C} / \mathrm{O}$ and $\mathrm{N} / \mathrm{O}$ ratios in gas and dust throughout our static disk, and to what extent they are affected by the presence of $\mathrm{CH}_{4}$ and $\mathrm{NH}_{3}$ over the full range of observed $\mathrm{CH}_{4}$ and $\mathrm{NH}_{3}$ abundances toward low-mass protostars. In this section we only consider pure ices, i.e. ices that are layered on a silicate mantle. In reality, the icy grain will have a layered structure with volatiles residing on top of a $\mathrm{H}_{2} \mathrm{O}$ ice substrate. However, if the volatile ice layer is thick enough and separated from the $\mathrm{H}_{2} \mathrm{O}$ ice layer, the interaction between $\mathrm{H}_{2} \mathrm{O}$ and the other volatile species will be minimal and thus the ices can be considered pure rather than water dominated (see also Section 1).
We explore the parameter space of possible $\mathrm{CH}_{4}$ abundances by assuming three different scenarios: (1) no $\mathrm{CH}_{4}$, (2) $\mathrm{CH}_{4}$-mid, and (3) the maximum $\mathrm{CH}_{4}$ observed abundance (hereafter $\mathrm{CH}_{4}$-max), $n_{\mathrm{CH}_{4}-\max }=0.13 \times n_{\mathrm{H}_{2} \mathrm{O}}$ (Öberg et al. 2008). Since the abundance of carbon grains is uncertain, we assume that all the carbon that is not in the form of $\mathrm{CH}_{4}, \mathrm{CO}$ and $\mathrm{CO}_{2}$ is in carbon grains, so that we reproduce the Solar $\mathrm{C} / \mathrm{O}$ ratio (gas+dust) of 0.54 .

Figure 2 shows the $\mathrm{C} / \mathrm{O}$ ratio in gas and dust as a function of semimajor axis in a static disk: no $\mathrm{CH}_{4}$ (top panel), $\mathrm{CH}_{4}$-mid (middle panel) and $\mathrm{CH}_{4}$-max (bottom panel). As in Öberg et al. (2011b) and Paper I, a gaseous $\mathrm{C} / \mathrm{O}$ ratio of unity can be achieved between the $\mathrm{CO}_{2}$ and $\mathrm{CO}$ snowlines, where oxygen gas is significantly depleted. The gas-phase $\mathrm{C} / \mathrm{O}$ ratio may be further enhanced between the $\mathrm{CO}_{2}$ and $\mathrm{CH}_{4}$ snowlines due to the presence of additional carbon gas from $\mathrm{CH}_{4}$. In this region, the $\mathrm{C} / \mathrm{O}$ ratio increases by $3 \%$ for $\mathrm{CH}_{4}$-mid and by $8 \%$ for $\mathrm{CH}_{4}$-max, as displayed in the middle and bottom panels of Figure 2. Based on the range of observed $\mathrm{CH}_{4}$ protostellar abundances, its presence in the disk only modestly affects the $\mathrm{C} / \mathrm{O}$ ratio.

We assume that the main nitrogen-bearing species are $\mathrm{N}_{2}$ and $\mathrm{NH}_{3}$, since other volatiles that contain nitrogen have significantly lower abundances in comparison (e.g., Mumma \& Charnley 2011). Similarly to the case of $\mathrm{CH}_{4}$, we explore the parameter space of possible $\mathrm{NH}_{3}$ abundances using observations toward low-mass protostars, 

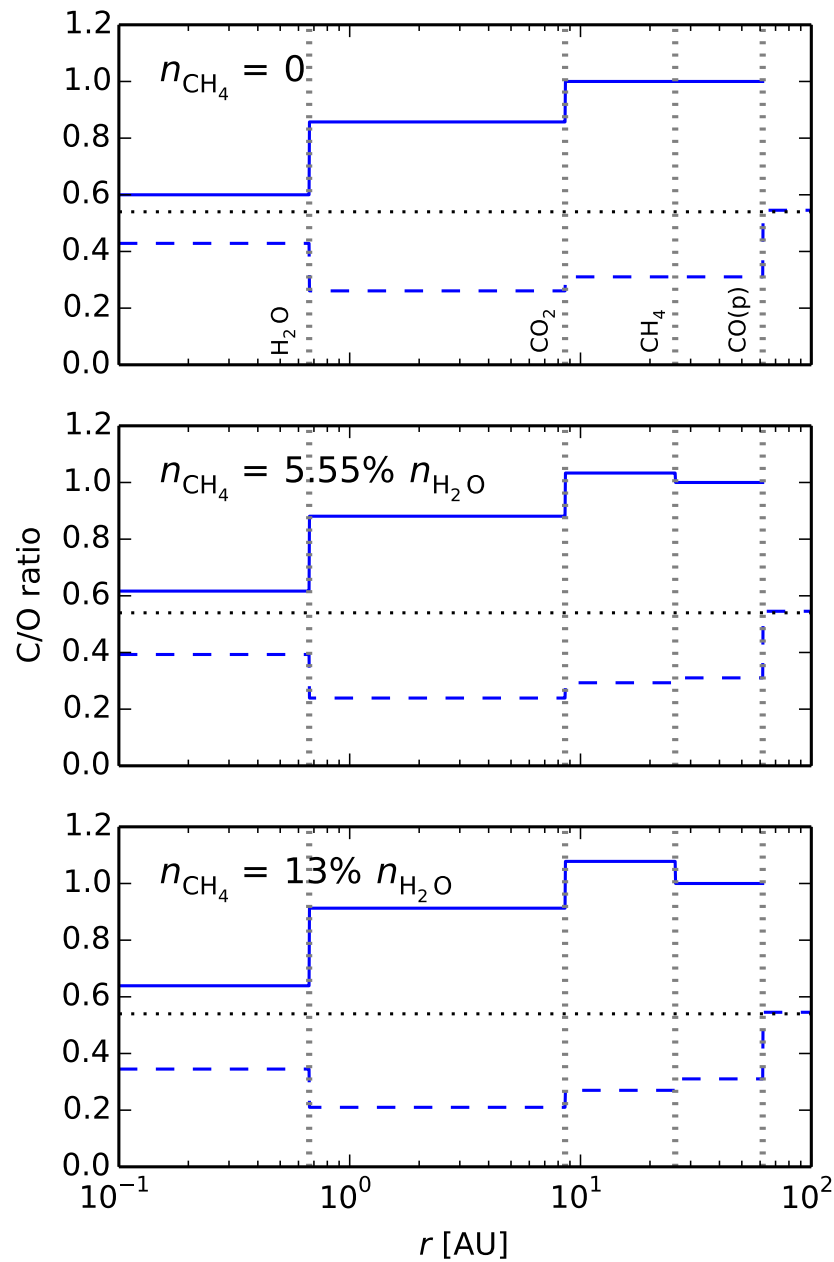

FIG. 2.- The $\mathrm{C} / \mathrm{O}$ ratio in gas (solid lines) and dust (dashed lines) as a function of semimajor axis in a static disk, assuming no carbon is present in the form of $\mathrm{CH}_{4}$ (top panel), the median observed $\mathrm{CH}_{4}$ abundance is assumed (middle panel), and the maximum observed $\mathrm{CH}_{4}$ abundance is assumed (bottom panel). The $\mathrm{C} / \mathrm{O}$ estimates are performed assuming that the $\mathrm{CO}$ ices are in pure form. The vertical dotted lines mark the snowline locations of the main $\mathrm{C}$ and $\mathrm{O}$ carriers. The horizontal dotted lines represent the stellar $\mathrm{C} / \mathrm{O}$ value. The presence of methane only modestly increases the $\mathrm{C} / \mathrm{O}$ ratio in gas between the $\mathrm{CO}_{2}$ and $\mathrm{CH}_{4}$ snowlines.

as follows: (1) no $\mathrm{NH}_{3}$, (2) $\mathrm{NH}_{3}$-mid, and (3) the maximum observed $\mathrm{NH}_{3}$ abundance $n_{\mathrm{NH}_{3}-\max }=0.15 \times n_{\mathrm{H}_{2} \mathrm{O}}$ (Bottinelli et al. 2010). In each case, the $\mathrm{N}_{2}$ abundance then simply follows as $n_{\mathrm{N}_{2}}=\left(n_{\mathrm{N}}-n_{\mathrm{NH}_{3}}\right) / 2$.

Figure 3 shows the snowline locations of the main oxygen and nitrogen carriers and the $\mathrm{N} / \mathrm{O}$ ratio in gas and dust as a function of semimajor axis in a static disk, for our three choices of the $\mathrm{NH}_{3}$ abundance: no $\mathrm{NH}_{3}$ (top panel), $\mathrm{NH}_{3}$-mid (middle panel) and $\mathrm{NH}_{3}$-max (bottom panel). For comparison, the horizontal dotted lines show the average $\mathrm{N} / \mathrm{O}$ ratio in the disk. As expected, the gaseous $\mathrm{N} / \mathrm{O}$ ratio generally exhibits an increasing trend towards the outer disk as more oxygen gas is depleted, with small decreases between the $\mathrm{NH}_{3}$ and $\mathrm{CO}_{2}$ snowlines (by $6 \%$ for $\mathrm{NH}_{3}$-mid and by $18 \%$ for $\mathrm{NH}_{3}$-max, respectively) due to $\mathrm{NH}_{3}$ freeze-out. While the presence of $\mathrm{NH}_{3}$ only moderately affects our results for the $\mathrm{N} / \mathrm{O}$ ratio, $\mathrm{NH}_{3}$ is important since otherwise the nitro-
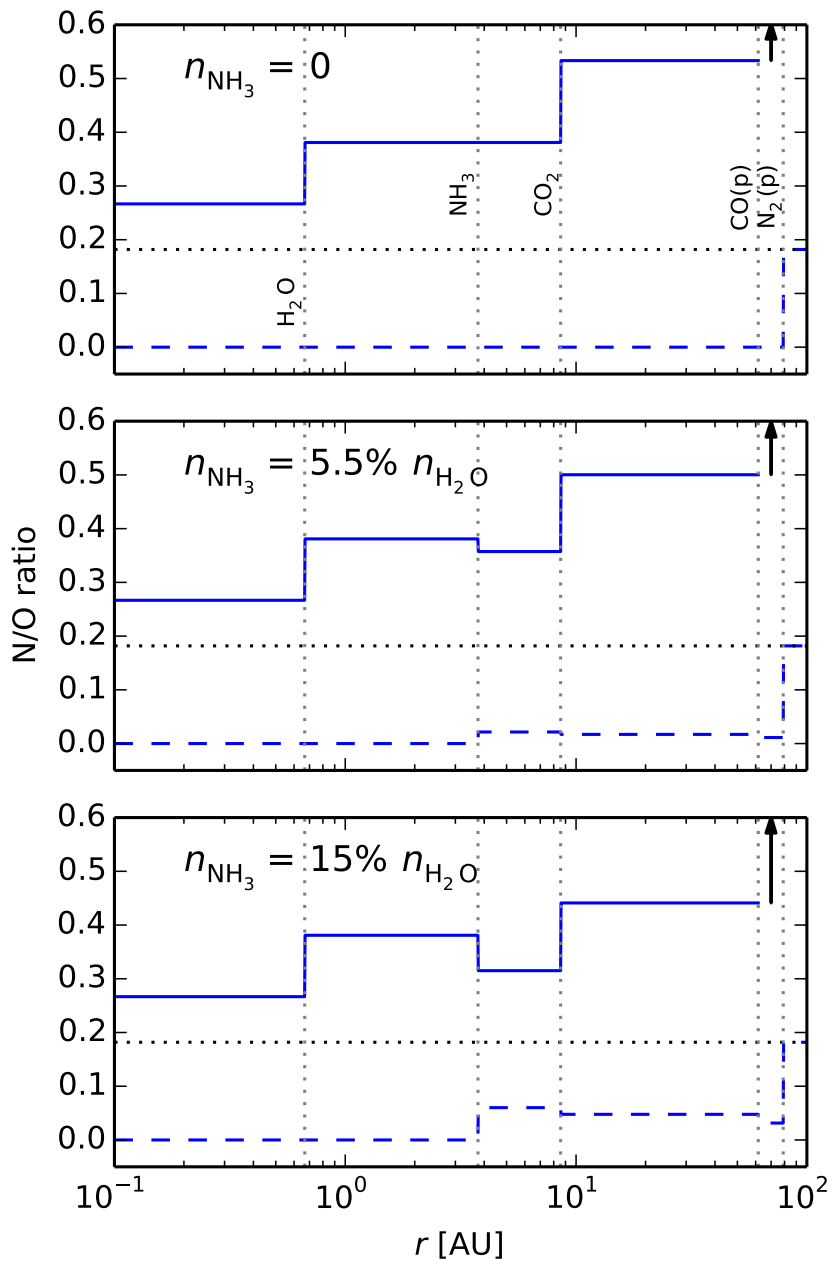

FIG. 3.- The N/O ratio in gas (solid lines) and dust (dashed lines) as a function of semimajor axis in a static disk, assuming no nitrogen is present in the form of $\mathrm{NH}_{3}$ (top panel), the median observed $\mathrm{NH}_{3}$ abundance is assumed (middle panel), and the maximum observed $\mathrm{NH}_{3}$ abundance is assumed (bottom panel). The $\mathrm{N} / \mathrm{O}$ estimates are performed assuming that the $\mathrm{CO}$ and $\mathrm{N}_{2}$ ices are in pure form. The vertical dotted lines mark the snowline locations of the main $\mathrm{C}, \mathrm{O}$ and $\mathrm{N}$ carriers. The horizontal dotted lines represent the average $\mathrm{N} / \mathrm{O}$ value in the disk. The gas-phase $\mathrm{N} / \mathrm{O}$ ratio is enhanced by a factor of two between the $\mathrm{H}_{2} \mathrm{O}$ and $\mathrm{CO}_{2}$ snowlines compared to its average value, and by a factor of three between the $\mathrm{CO}_{2}$ and $\mathrm{CO}$ snowlines. The arrows mark a highly elevated $\mathrm{N} / \mathrm{O}$ ratio in gas between the $\mathrm{CO}$ and $\mathrm{N}_{2}$ snowlines due to the depletion of oxygen gas in this region. The presence of $\mathrm{NH}_{3}$ moderately decreases the $\mathrm{N} / \mathrm{O}$ ratio in gas between the $\mathrm{NH}_{3}$ and $\mathrm{CO}_{2}$ snowlines.

gen content in solid bodies would be more depleted than is observed for comets and asteroids (Wyckoff et al. 1991, Mumma \& Charnley 2011, Bergin et al. 2015).

The gas-phase $\mathrm{N} / \mathrm{O}$ ratio is enhanced by a factor of two outside the $\mathrm{H}_{2} \mathrm{O}$ snowline compared to its average value, by more than a factor of three between the $\mathrm{CO}_{2}$ and $\mathrm{CO}$ snowlines, and by orders of magnitude between the $\mathrm{CO}$ and $\mathrm{N}_{2}$ snowlines. This latter region can span tens of $\mathrm{AU}$ depending on disk parameters and the relative $\mathrm{CO}$ and $\mathrm{N}_{2}$ ice binding environment. This $\mathrm{N} / \mathrm{O}$ enhancement is more pronounced than the $\mathrm{C} / \mathrm{O}$ gas phase enhancement of a factor of two in the outer disk (see Figure 2 ). 


\section{3. $C / N / O$ Ratios in Dynamic Disks}

Here we use the model of Section 2 to estimate the movement of the $\mathrm{CO}$ and $\mathrm{N}_{2}$ snowlines for different grain morphologies in a viscous disk. Figure 4 shows the $\mathrm{H}_{2} \mathrm{O}$, $\mathrm{CO}_{2}$ and $\mathrm{CO}$ snowline locations for particles with initial sizes $\sim 0.05 \mathrm{~cm} \lesssim s \lesssim 7 \mathrm{~m}$ as well as estimates for the $\mathrm{C} / \mathrm{O}$ ratio in gas and dust in a viscous disk, with the $\mathrm{CO}$ snowline calculated under different grain morphologies as noted above. We assume there is no carbon in the form of $\mathrm{CH}_{4}$. The true snowline for particles that desorb outside the static snowline is the static snowline itself, hence desorbing particles with $s<0.05 \mathrm{~cm}$ do not form true snowlines. If the $\mathrm{CO}$ binding environment is known, the CO snowline moves inward by up to 50\% compared to a static disk for each case (pure and water dominated ices) due to disk dynamics. The full range of potential $\mathrm{CO}$ snowlines taking into account both ice compositions and disk dynamics span $\sim 8.7$ AU to $\sim 61 \mathrm{AU}$, which is a factor of $\sim 7$ difference. This implies that gas phase $\mathrm{C} / \mathrm{O}$ ratios of order unity may be reached in the giant planet forming zone, and the $\mathrm{CO}$ snowline may be inside $10 \mathrm{AU}$ for certain disk parameters.

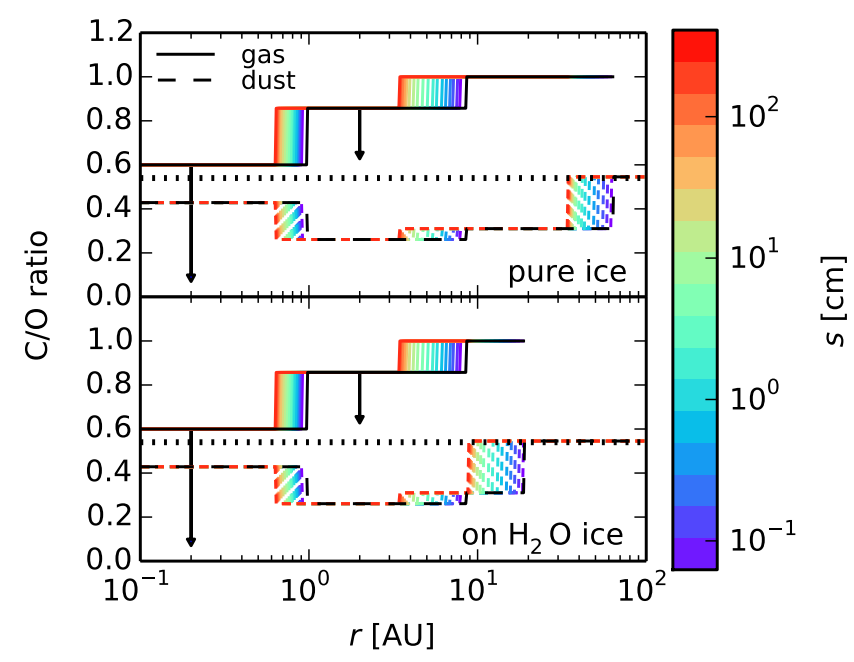

FIG. 4.- C/O ratio estimates in gas (solid lines) and dust (dashed lines) as function of semimajor axis in a viscous disk, for $\mathrm{CO}$ as pure ice (top panel) or as water dominated ices (bottom panel). The $\mathrm{H}_{2} \mathrm{O}, \mathrm{CO}_{2}$ and $\mathrm{CO}$ snowlines are shown for particles with initial sizes $\sim 0.05 \mathrm{~cm} \lesssim s \lesssim 7 \mathrm{~m}$ as indicated by the color bar. $\mathrm{The} \mathrm{C} / \mathrm{O}$ ratio in a static disk (black lines) is shown for comparison. The arrows show that the $\mathrm{C} / \mathrm{O}$ ratio in gas will decrease inside the $\mathrm{H}_{2} \mathrm{O}$ and $\mathrm{CO}_{2}$ snowlines in the viscous disk, as the relative fluxes of the desorbed icy particles and the overall nebular gas will cause an excess of oxygen gas inside these snowlines (see Paper I for details). The presence of $\mathrm{CO}$ in a water ice environment rather than as pure ice moves the CO snowline significantly inward by $\sim 70 \%$. Taken together, disk dynamics and ice compositions move the CO snowline inward by a factor of $\sim 7$.

Figure 5 shows the $\mathrm{H}_{2} \mathrm{O}, \mathrm{CO}_{2}, \mathrm{CO}$ and $\mathrm{N}_{2}$ snowline locations in a viscous disk for the same range of initial particle sizes as in Figure 4, and with the $\mathrm{CO}$ and $\mathrm{N}_{2}$ snowlines calculated assuming different grain morphologies as explained above, as well as estimates for the $\mathrm{N} / \mathrm{O}$ ratio throughout the disk. For simplicity, we assume that all nitrogen is the form of $\mathrm{N}_{2}$. This choice is justified since the presence of some $\mathrm{NH}_{3}$ only moderately changes the $\mathrm{N} / \mathrm{O}$ ratio (see Figure 3), and since we are primarily interested in the $\mathrm{N}_{2}$ snowline locations rather than exact values for the $\mathrm{N} / \mathrm{O}$ ratio. The innermost $\mathrm{N}_{2}$ snowlines in the viscous disk, created by particles with $s \sim 7 \mathrm{~m}$ for our fiducial model, are located at $r_{\mathrm{N}_{2} \text {,pure }} \approx 42 \mathrm{AU}$ for $\mathrm{N}_{2}$ as pure ice and at $r_{\mathrm{N}_{2} \text {,water }} \approx 11 \mathrm{AU}$ for $\mathrm{N}_{2}$ in water dominated ices. Thus for each case (pure versus water dominated ices), the $\mathrm{N}_{2}$ snowline moves inward by up to $50 \%$ due to disk dynamics. By taking into account both ice compositions and disk dynamics, the full range of potential $\mathrm{N}_{2}$ snowlines span $\sim 11$ to $\sim 79 \mathrm{AU}$, which is a factor of $\sim 7$ difference. Similarly to the case for $\mathrm{CO}$, the $\mathrm{N}_{2}$ snowline may be close to $10 \mathrm{AU}$ for certain disk models.

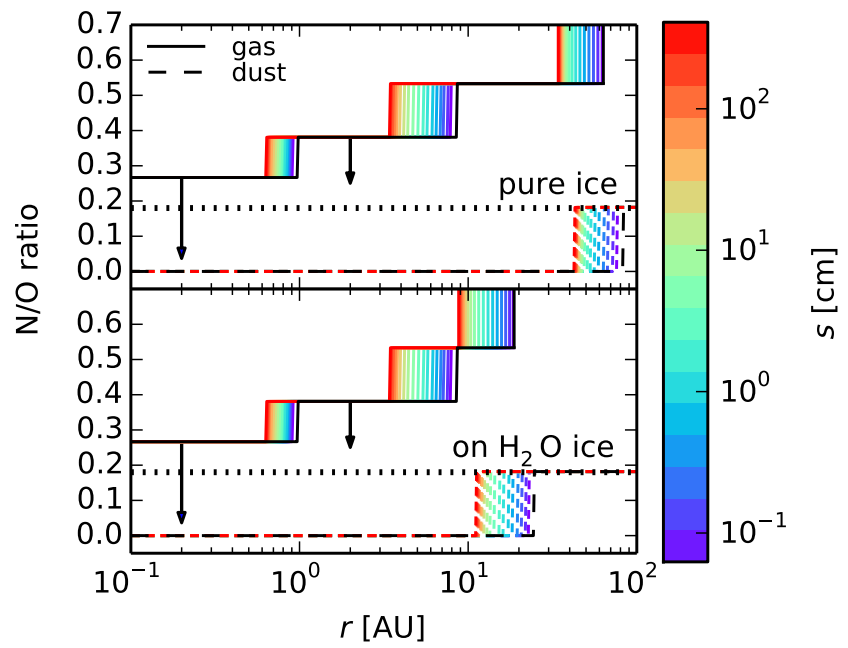

FIG. 5.- N/O ratio estimates in gas (solid lines) and dust (dashed lines) as function of semimajor axis in a viscous disk, for $\mathrm{CO}$ and $\mathrm{N}_{2}$ as pure ices (top panel) or as water dominated ices (bottom panel). The $\mathrm{H}_{2} \mathrm{O}, \mathrm{CO}_{2}, \mathrm{CO}$ and $\mathrm{N}_{2}$ snowlines are shown for particles with initial sizes $\sim 0.05 \mathrm{~cm} \lesssim s \lesssim 7 \mathrm{~m}$ as indicated by the color bar. The $\mathrm{N} / \mathrm{O}$ ratio in a static disk (black lines) is shown for comparison. The arrows show that the $\mathrm{N} / \mathrm{O}$ ratio in gas will decrease inside the $\mathrm{H}_{2} \mathrm{O}$ and $\mathrm{CO}_{2}$ snowlines in the viscous disk, as the relative fluxes of the desorbed icy particles and the overall nebular gas will cause an excess of oxygen gas inside these snowlines (see Paper I for details). Radial drift and gas accretion move the $\mathrm{N}_{2}$ snowline inward by up to $\sim 50 \%$ compared to a static disk. The presence of $\mathrm{N}_{2}$ in a water ice environment rather than as pure ice moves the $\mathrm{N}_{2}$ snowline significantly inward by $\sim 70 \%$. Taken together, disk dynamics and ice compositions move the $\mathrm{N}_{2}$ snowline inward by a factor of $\sim 7$. The results of an enhanced gas-phase $\mathrm{N} / \mathrm{O}$ ratio between the $\mathrm{H}_{2} \mathrm{O}$ and $\mathrm{CO}$ snowlines compared to its average value, and of highly elevated $\mathrm{N} / \mathrm{O}$ ratios in gas between the $\mathrm{CO}$ and $\mathrm{N}_{2}$ snowlines (see Figure 3, are preserved.

\section{DISCUSSION}

This study shows that the gas-phase N/O ratio in protoplanetary disks is considerably enhanced throughout most of the disk midplane compared to its average value. As demonstrated in Figure 6. the gaseous $\mathrm{N} / \mathrm{O}$ ratio is enhanced by a factor of two beyond the $\mathrm{H}_{2} \mathrm{O}$ snowline, by more than a factor of three between the $\mathrm{CO}_{2}$ and $\mathrm{CO}$ snowlines, and by several orders of magnitude between the $\mathrm{CO}$ and $\mathrm{N}_{2}$ snowlines. Thus constraining the $\mathrm{N} / \mathrm{O}$ ratio in a giant planet atmosphere could be used to trace its formation origins. 
Theoretical models of the magnitude and role of $\mathrm{N} / \mathrm{O}$ (and N/C) ratios in exoplanet atmospheres are needed in order to use these ratios as probes for a planet's formation location. Models that explore the effect of varying the $\mathrm{C} / \mathrm{O}$ ratio in exoplanet atmospheres exist in literature, and they display a large and observable effect on gas giant envelope chemistry (Lodders 2009, Mollière et al. 2015). However, no similar model explorations exist for the effect of $\mathrm{N} / \mathrm{O}$ and $\mathrm{C} / \mathrm{N} / \mathrm{O}$ ratios, and both are needed to exploit this potential constraint. Given the existence of such theoretical models, measurements of the N/O ratio in planetary envelopes may be possible to infer from atmospheric compositions of nitrogen versus carbon and oxygen bearing species. Nitrogen carriers have not been targeted so far due to lack of instrument sensitivity, but such observations and detections are likely in the near future with the advent of JWST (e.g., $\mathrm{NH}_{3}$, Greene et al. 2016). The $\mathrm{N} / \mathrm{O}$ ratio enhancement is larger than that of the gas phase $\mathrm{C} / \mathrm{O}$ ratio throughout most of the disk. Thus measurements of an enhanced $\mathrm{C} / \mathrm{O}$ ratio in an exoplanet atmosphere could be corroborated (disproved) by measurements of enhanced (non-enhanced) N/O ratios. Moreover, Figure 6 shows that giant planets that have formed in situ between the $\mathrm{H}_{2} \mathrm{O}$ and $\mathrm{CO}$ snowlines are expected to present elevated both $\mathrm{C} / \mathrm{O}$ and $\mathrm{N} / \mathrm{O}$ ratios in their atmospheres, whereas planets between the $\mathrm{CO}$ and $\mathrm{N}_{2}$ snowlines will have a highly enhanced $\mathrm{N} / \mathrm{O}$ ratio in their atmospheres, but not $\mathrm{C} / \mathrm{O}$.

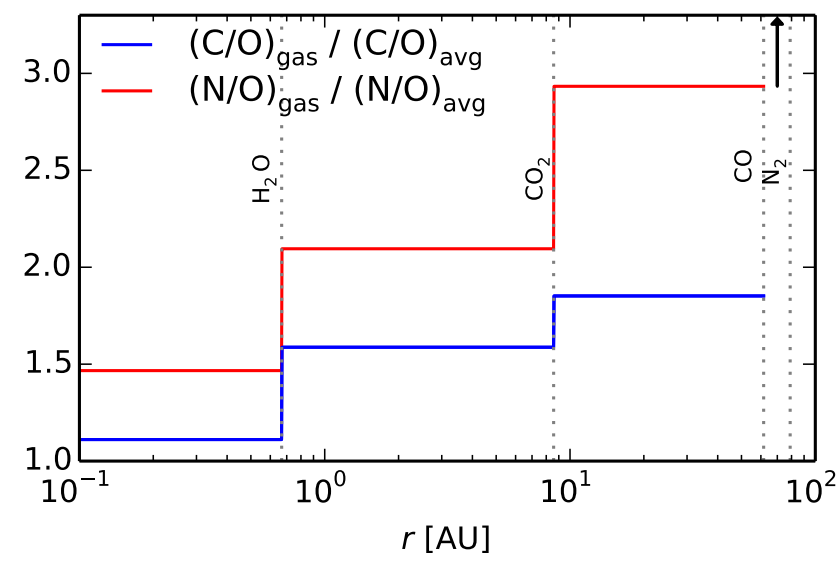

Fig. 6. - Gas phase C/O (blue curve) and N/O (red curve) ratios divided by the average $\mathrm{C} / \mathrm{O}$ and $\mathrm{N} / \mathrm{O}$ ratio in a static disk, assuming $\mathrm{CO}$ and $\mathrm{N}_{2}$ are pure ices, and there is no $\mathrm{CH}_{4}$ or $\mathrm{NH}_{3}$. The dashed vertical lines mark the $\mathrm{H}_{2} \mathrm{O}, \mathrm{CO}_{2}, \mathrm{CO}$ and $\mathrm{N}_{2}$ snowlines. The arrow indicates that the $\mathrm{N} / \mathrm{O}$ ratio is enhanced by orders of magnitude compared to its average value between the $\mathrm{CO}$ and $\mathrm{N}_{2}$ snowlines. The gaseous $\mathrm{N} / \mathrm{O}$ ratio is enhanced throughout most of the disk, and more enhanced than the $\mathrm{C} / \mathrm{O}$ ratio.

Due to disk dynamics and ice compositions, the locations of the $\mathrm{CO}$ and $\mathrm{N}_{2}$ snowlines, and thus the disk regions with highly elevated gas phase $\mathrm{N} / \mathrm{O}$ and $\mathrm{C} / \mathrm{O}$ ratios, are uncertain and may span tens of $\mathrm{AU}$. Both ice morphologies discussed in this study, pure and water dominated ices, are plausible in protoplanetary disks and depend on whether $\mathrm{H}_{2} \mathrm{O}$ and $\mathrm{CO}$ ices formed on similar timescales or successively (e.g., Garrod \& Pauly 2011). Observations of protostellar cores show that a large fraction of CO is bound in a pure ice multilayer (Pontoppidan et al. 2003), but theoretical models also suggest an icy mantle structure where $\mathrm{CO}$ resides on a $\mathrm{H}_{2} \mathrm{O}$ ice layer (e.g., Collings et al. 2003). One can also imagine a scenario where $\mathrm{CO}$ is in a water binding environment and $\mathrm{N}_{2}$ is not. This could be attributed to the fact that $\mathrm{H}_{2} \mathrm{O}$ may bind preferentially to $\mathrm{CO}$ than $\mathrm{N}_{2}$, since both $\mathrm{H}_{2} \mathrm{O}$ and $\mathrm{CO}$ are polar molecules while $\mathrm{N}_{2}$ is not. It is also possible for $\mathrm{N}_{2}$ ices to form later than $\mathrm{CO}$ (e.g., Pagani et al.2012), and thus be deposited on the outer layers of the icy mantles which are typically water poor (e.g., Garrod \& Pauly 2011). The impact of the ice environment on the snowline location is much smaller in the case of $\mathrm{CO}_{2}$ and $\mathrm{NH}_{3}$, as their binding energies and behavior are closer to that of $\mathrm{H}_{2} \mathrm{O}$. No detailed measurements for the $\mathrm{CH}_{4}$ binding energy in a water environment exist so far, but due to its low desorption temperature a similar behavior to that of $\mathrm{CO}$ and $\mathrm{N}_{2}$ would be expected. While the presence of some carbon in the form of $\mathrm{CH}_{4}$ only modestly affects our results, $\mathrm{CH}_{4}$ may become important in disks where a large fraction of the $\mathrm{CO}$ abundance has been converted into hydrocarbons (e.g., Du et al. 2015).

Changes in stellar luminosity (e.g., Kennedy et al. 2006) and gas mass accretion rate (e.g., Chambers 2009), as well as the evolution of icy dust particles due to grain growth and fragmentation (e.g., Birnstiel et al. 2012), may introduce additional uncertainties in the snowline locations, and thus the $\mathrm{C} / \mathrm{N} / \mathrm{O}$ ratios. Moreover, the diffusion of vapor across the snowlines following the cold finger effect (Stevenson \& Lunine 1988, Cyr et al. 1998) will change the shape of the $\mathrm{C} / \mathrm{O}$ and $\mathrm{N} / \mathrm{O}$ curves and therefore the magnitude of the $\mathrm{C} / \mathrm{N} / \mathrm{O}$ ratios between different snowlines. The effect of dynamical processes on snowline locations is discussed in more detail in Paper I, Section 5.2. Given the number of uncertainties in snowline locations, detections of snowlines in a sample of disks at different evolutionary stages are needed to provide observational constraints on the relative importance of ice compositions and disk dynamics in setting snowline locations. The uncertainties in snowline locations caused by disk dynamics, ice compositions, and other effects outlined above can be resolved in extreme cases, such as a detection of a $\mathrm{CO}$ snowline at a temperature corresponding to pure $\mathrm{CO}$ ice desorption in a static disk (e.g., Qi et al. 2013 at $\sim 17 \mathrm{~K}$ ) or $\mathrm{CO}$ desorption from a water dominated ice in a dynamic disk. In intermediate cases it is more difficult to resolve the relative importance of ice compositions and disk dynamics. For example, the CO snowline in HD 163296 is at a higher temperature of $\sim 25 \mathrm{~K}$ (Qi et al. 2015), which could be caused either by $\mathrm{CO}$ being in a water dominated environment or by dynamical effects that push the CO snowline inward. Detections of multiple snowlines in the same disk could potentially break this degeneracy.

Uncertainties in snowline locations of this magnitude also affect interpretations of Solar system observations. Recent measurements of nitrogen abundance in comet $67 \mathrm{P} /$ Churyumov-Gerasimenko found a $\mathrm{N}_{2} / \mathrm{CO}$ ratio $\sim$ $10^{-3}$ (Rubin et al. 2015). A low $\mathrm{N}_{2} / \mathrm{CO}$ ratio is consistent with comets having formed inside the $\mathrm{N}_{2}$ snowline where $\mathrm{N}_{2}$ is still in the gas phase. However, it is also possible that the measured $\mathrm{N}_{2}$ abundance in $67 \mathrm{P}$ may be due to post-formation processes such as radiogenic heating (Rubin et al. 2015), and thus may not reflect 
the comet's primordial composition. Theoretical models suggest that Jupiter-family comets, such as 67P, originate from the Kuiper belt (Duncan \& Levison 1997; but see Rubin et al. 2015 for alternative formation scenarios for $67 \mathrm{P}$ ). It is thus possible, in principle, to use measurements of the $\mathrm{N}_{2}$ abundance in Jupiter-family comets to determine where the $\mathrm{N}_{2}$ snowline was located in our Solar system. However due to the uncertainty in the calculated location of the $\mathrm{N}_{2}$ snowline (see Section 3.3), as well as the uncertainty of the formation zone of Jupiter-family comets (anywhere between 5 and >30 AU; Pontoppidan et al. 2014), more detailed modeling is needed.

\section{SUMMARY}

In this paper we explore the role of icy grain compositions and disk dynamics on the snowline locations of major volatile carrier molecules and the $\mathrm{C} / \mathrm{N} / \mathrm{O}$ ratios in protoplanetary disks. We enhance the coupled drift-desorption model developed in Piso et al. (2015) by adding more carbon- and nitrogen-bearing species into our framework, and by considering different binding ice environments. Our results can be summarized as follows:

1. Due to the high volatility of $\mathrm{N}_{2}$, the gaseous $\mathrm{N} / \mathrm{O}$ ratio outside the $\mathrm{H}_{2} \mathrm{O}$ snowline is enhanced by a factor of two compared to its average value, by more than a factor of three between the $\mathrm{CO}_{2}$ and $\mathrm{CO}$ snowlines, and by many orders of magnitude between the $\mathrm{CO}$ and $\mathrm{N}_{2}$ snowlines due to the complete depletion of oxygen gas in this region. This enhancement is more pronounced than in the case of the gas-phase $\mathrm{C} / \mathrm{O}$ ratio, which is increased by at most a factor of two compared to the stellar value.

2. The effect of $\mathrm{CH}_{4}$ and $\mathrm{NH}_{3}$ on the $\mathrm{C} / \mathrm{O}$ and $\mathrm{N} / \mathrm{O}$ ratios is small, even when we consider the maximum observed $\mathrm{CH}_{4}$ and $\mathrm{NH}_{3}$ abundances in protostellar cores. In this scenario, the gas phase $\mathrm{C} / \mathrm{O}$ ratio increases by $8 \%$ between the $\mathrm{CO}_{2}$ and $\mathrm{CH}_{4}$ snowlines, and the gaseous $\mathrm{N} / \mathrm{O}$ ratio decreases by $18 \%$ between the $\mathrm{NH}_{3}$ and $\mathrm{CO}_{2}$ snowlines. In both cases, large gas phase $\mathrm{C} / \mathrm{O}$ and $\mathrm{N} / \mathrm{O}$ ratios in the outer disk are preserved.

3. Grain composition sensitively affects the $\mathrm{CO}$ and $\mathrm{N}_{2}$ snowline locations. If $\mathrm{CO}$ and $\mathrm{N}_{2}$ reside in water dominated rather than pure ices, their snowlines move inward by up to $\sim 70 \%$. This effect is separate from that of radial drift and viscous gas accretion, which also cause an inward movement of the $\mathrm{CO}$ and $\mathrm{N}_{2}$ snowlines by up to $\sim 50 \%$.

4. The locations of the $\mathrm{CO}$ and $\mathrm{N}_{2}$ snowlines are uncertain when we consider both viscous versus static disks, and pure versus water dominated ices. The snowlines in a viscous disk with $\mathrm{CO}$ or $\mathrm{N}_{2}$ in a water environment are by up to a factor of $\sim 7$ closer to the host star that in a static disk with $\mathrm{CO}$ or $\mathrm{N}_{2}$ as pure ices.

Our results have direct consequences for the composition of nascent giant planets. The considerable inward movement of the $\mathrm{CO}$ and $\mathrm{N}_{2}$ snowlines due to the ice grains being water dominated rather than pure ices implies than giant planets with high $\mathrm{C} / \mathrm{O}$ and/or $\mathrm{N} / \mathrm{O}$ ratios in their atmospheres may form closer in than previously predicted by theoretical models. Moreover, our model shows that wide separation gas giants may have an excess of nitrogen in their envelopes, which may be used to trace their origins.

We thank the anonymous referee for helpful comments and suggestions. This work is supported by a Simons Collaboration on the Origins of Life (SCOL) investigator award to KIO. JP was supported by the Banneker institute.

\section{REFERENCES}

Ali-Dib, M., Mousis, O., Petit, J.-M., \& Lunine, J. I. 2014, ApJ, 785,125

Andrews, S. M., Wilner, D. J., Hughes, A. M., Qi, C., \& Dullemond, C. P. 2010, ApJ, 723, 1241

Atreya, S. K., Donahue, T. M., \& Kuhn, W. R. 1978, Science, 201,611

Bergin, E. A., Blake, G. A., Ciesla, F., Hirschmann, M. M., \& Li, J. 2015, Proceedings of the National Academy of Science, 112, 8965

Birnstiel, T., Klahr, H., \& Ercolano, B. 2012, A\&A, 539, A148

Bisschop, S. E., Fraser, H. J., Öberg, K. I., van Dishoeck, E. F., \& Schlemmer, S. 2006, A\&A, 449, 1297

Boogert, A. C. A., Gerakines, P. A., \& Whittet, D. C. B. 2015, ARA\&A, 53, 541

Bottinelli, S., Boogert, A. C. A., Bouwman, J., et al. 2010, ApJ, 718,1100

Chambers, J. E. 2009, ApJ, 705, 1206

Chiang, E., \& Youdin, A. N. 2010, Annual Review of Earth and Planetary Sciences, 38, 493

Collings, M. P., Anderson, M. A., Chen, R., et al. 2004, MNRAS, 354,1133

Collings, M. P., Dever, J. W., Fraser, H. J., \& McCoustra, M. R. S. 2003, Ap\&SS, 285, 633

Cyr, K. E., Sears, W. D., \& Lunine, J. I. 1998, Icarus, 135, 537 Du, F., Bergin, E. A., \& Hogerheijde, M. R. 2015, ArXiv e-prints, arXiv: 1506.03510

Duncan, M. J., \& Levison, H. F. 1997, Science, 276, 1670

Fayolle, E. C., Balfe, J., Loomis, R., et al. 2016, ApJ, 816, L28

Fraser, H. J., Collings, M. P., McCoustra, M. R. S., \& Williams, D. A. 2001, MNRAS, 327, 1165

Garrod, R. T., \& Herbst, E. 2006, A\&A, 457, 927
Garrod, R. T., \& Pauly, T. 2011, ApJ, 735, 15

Greene, T. P., Line, M. R., Montero, C., et al. 2016, ApJ, 817, 17

Henning, T., \& Semenov, D. 2013, Chemical Reviews, 113, 9016

Hollenbach, D., Kaufman, M. J., Bergin, E. A., \& Melnick, G. J. 2009, ApJ, 690, 1497

Kennedy, G. M., Kenyon, S. J., \& Bromley, B. C. 2006, ApJ, 650, L139

Lectez, S., Simon, J.-M., Mousis, O., et al. 2015, ApJ, 805, L1

Lodders, K. 2003, ApJ, 591, 1220

—. 2009, ArXiv e-prints, arXiv:0910.0811

Madhusudhan, N., Amin, M. A., \& Kennedy, G. M. 2014, ApJ, 794, L12

Mandell, A. M., Bast, J., van Dishoeck, E. F., et al. 2012, ApJ, 747,92

Mandt, K. E., Mousis, O., Lunine, J., \& Gautier, D. 2014, ApJ, 788, L24

Martín-Doménech, R., Muñoz Caro, G. M., Bueno, J., \& Goesmann, F. 2014, A\&A, 564, A8

Mollière, P., van Boekel, R., Dullemond, C., Henning, T., \& Mordasini, C. 2015, ApJ, 813, 47

Mousis, O., Fletcher, L. N., Lebreton, J.-P., et al. 2014, Planet. Space Sci., 104, 29

Mousis, O., Lunine, J. I., Luspay-Kuti, A., et al. 2016, ApJ, 819, L33

Mumma, M. J., \& Charnley, S. B. 2011, ARA\&A, 49, 471

Noble, J. A., Congiu, E., Dulieu, F., \& Fraser, H. J. 2012, MNRAS, 421, 768

Öberg, K. I., Boogert, A. C. A., Pontoppidan, K. M., et al. 2008, ApJ, 678, 1032

-. 2011a, ApJ, 740, 109 
Öberg, K. I., Murray-Clay, R., \& Bergin, E. A. 2011b, ApJ, 743, L16

Öberg, K. I., van Broekhuizen, F., Fraser, H. J., et al. 2005, ApJ, 621, L33

Owen, T., Mahaffy, P. R., Niemann, H. B., Atreya, S., \& Wong, M. 2001, ApJ, 553, L77

Pagani, L., Bourgoin, A., \& Lique, F. 2012, A\&A, 548, L4

Pérez, L. M., Carpenter, J. M., Chandler, C. J., et al. 2012, ApJ, 760, L17

Piso, A.-M. A., Öberg, K. I., Birnstiel, T., \& Murray-Clay, R. A. 2015, ApJ, 815, 109

Pontoppidan, K. M., Salyk, C., Bergin, E. A., et al. 2014 Protostars and Planets VI, 363

Pontoppidan, K. M., Fraser, H. J., Dartois, E., et al. 2003, A\&A, 408, 981

Qi, C., Öberg, K. I., Andrews, S. M., et al. 2015, ApJ, 813, 128

Qi, C., Öberg, K. I., Wilner, D. J., et al. 2013, Science, 341, 630
Rodgers, S. D., \& Charnley, S. B. 2002, MNRAS, 330, 660

Rubin, M., Altwegg, K., Balsiger, H., et al. 2015, Science, 348, 232

Schegerer, A. A. \& Wolf, S. 2010, A\&A, 517, A87

Shakura, N. I., \& Sunyaev, R. A. 1973, A\&A, 24, 337

Stevenson, D. J., \& Lunine, J. I. 1988, Icarus, 75, 146

van Dishoeck, E. F. 2006, Proceedings of the National Academy of Science, 103, 12249

van Dishoeck, E. F., Bergin, E. A., Lis, D. C., \& Lunine, J. I. 2014, Protostars and Planets VI, 835

Visser, R., van Dishoeck, E. F., Doty, S. D., \& Dullemond, C. P. 2009, A\&A, 495, 881

Williams, D., \& Herbst, E. 2002, Surface Science, 500, 823

Wyckoff, S., Tegler, S. C., \& Engel, L. 1991, ApJ, 367, 641

Zhang, K., Pontoppidan, K. M., Salyk, C., \& Blake, G. A. 2013, ApJ, 766, 82 